\title{
Empresas madeireiras e resíduos sólidos na Região Metropolitana de Belém
}

\section{Industries timber and solid residues in the Metropolitan area of Belém}

Wilson Fernandes Ramos - Engenheiro agrônomo pela Universidade Federal Rural da Amazônia (UFRA). Mestre em Ciências Ambientais pelo Programa de Pós-Graduação em Ciências Ambientais (PPGCA) da UFPA. E-mail: wilson.f.ramos18@hotmail.com

Gilma Irabel Rego D'Aquino - Socióloga. Analista de Ciência e Tecnologia. Museu Paraense Emilio Goedi/MCTI, Belém (PA). gilma@museu-goeldi.br

Maria de Lourdes Pinheiro Ruivo - Doutora em Agronomia pela Universidade Federal de Viçosa/MG. Mestre em Agronomia pela Universidade Federal Rural da Amazônia/ UFRA. Bolsista do CNPQ. E-mail: ruivo@museu-goeldi.br

\begin{abstract}
Resumo
O presente artigo apresenta informações preliminares sobre o levantamento da quantidade de indústrias de base florestal que atuam na Região Metropolitana de Belém (RMB) e o potencial em relação aos resíduos gerados por essas indústrias. Para a coleta de dados, foram utilizados os bancos de dados da Secretaria Estadual do Meio Ambiente e da Associação das Indústrias Exportadoras de Madeira do Estado do Pará. Os resultados preliminares indicam que 120 indústrias do setor madeireiro são, potencialmente, geradoras de resíduos madeireiros na RMB. Os resíduos gerados por essas indústrias acarretam inúmeros problemas de ordem social e ambiental em virtude de não haver uma política de reaproveitamento desse material. Muito desses resíduos gerados na RMB são doados para fábricas de cerâmicas, olarias ou reciclados como biomassa para a geração de energia térmica, a qual é usada na própria empresa.
\end{abstract}

\section{Palavras-chave}

Indústria de base florestal. Resíduo madeireiro. Problemas socioambientais.

\begin{abstract}
This paper presents preliminary information about raising the amount of forest-based industries that operate in the metropolitan area of Belém (RMB) and the potential for wastes generated by these industries. To collect data, the databases of the State Secretary of the Environment and the Association of Industries Exporters of Pará Wood were used. Preliminary results indicate that 120 industries in the timber sector are potentially wood waste generators in RMB. The waste generated by these industries entails many problems of social and environmental as they do not have a political reuse of this material. Much of this waste generated in $\mathrm{RMB}$ are donated to factories of ceramics, pottery or recycled as biomass to generate thermal energy, which is used in the industry itself.
\end{abstract}

\section{Keywords}

Forest-based industry. Wood residue. Social and environmental problems. 


\section{INTRODUÇÃO}

O presente artigo apresenta informações preliminares sobre o número de empresas madeireiras que atuam na Região Metropolitana de Belém (RMB) e acerca da geração e aproveitamento dos resíduos destas indústrias. Estas informações são relevantes, visto que a indústria de base florestal para a RMB e principalmente para o Estado do Pará é uma das atividades econômicas mais importantes, juntamente com a mineração e a agropecuária (FERREIRA et al. 2005; VERÍSSIMO et al. 2006). Porém, é também uma atividade que gera considerável quantidade de resíduos sólidos madeireiros (CEQUEIRO et al. 2012; BATISTA et al. 2013).

Os dados sobre as empresas do setor de base florestal da RMB foram levantados a Secretaria Estadual do Meio Ambiente (SEMA), que é o órgão responsável pelas questões legais relacionadas ao meio ambiente no Estado do Pará, e também de informações sobre o setor madeireiro obtidas por meio do acesso ao site da Associação das Indústrias Exportadoras de Madeira do Estado do Pará (AIMEX), de novembro de 2014 a janeiro de 2015.

Ao analisar as características das empresas em estudo, observou-se que a SEMA apresenta empresas que comercializam produtos acabados, fazem o desdobro da madeira, beneficiam, armazenam e usam de outras formas a matéria-prima madeira; enquanto as informações que constam no banco de dados da AIMEX indicam empresas de processamento da madeira em linha, compensados, madeira aparelhada, pisos de madeira, lâminas torneadas, forros, portas, decks, etc. Entretanto, a grande maioria trabalha com madeira serrada e compensado. Sendo assim, consideradas empresas potencialmente geradoras de resíduos, o que pode acarretar problemas ambientais e de saúde pública dos trabalhadores destas empresas e moradores vizinhos, o que já é registrado na literatura (LELIS, 2001; ZENID, 2009; HASAN et al. 2011; RIUL; RIBEIRO, 2012).

A partir dos dados coletados na AIMEX e na SEMA, foi feito o cruzamento dessas informações. Neste processo, verificou-se que a maioria das empresas constantes no site da AIMEX constavam também na lista fornecida pela SEMA. No levantamento das empresas localizadas na Região Metropolitana de Belém, feito na SEMA, foi contabilizada uma população de 556 empresas dos mais variados ramos, como comércio, serrarias, beneficiadoras de madeira, as que armazenam e as que consomem os produtos de madeireiros de alguma forma. Sendo que o órgão divide essas informações por "status", com isso foram selecionadas apenas as que tinham "status" ativo, excluindo, no primeiro 
momento, as que tinham seu "status" suspenso, suprimido, excluído e reprovado (Quadro 1). Dessa maneira, o número de empresas reduziu, chegando ao total de 257 empresas ativas.

Quadro 1 - Descrição do Número de empresas fornecidas pela SEMA e a descrição dos "status".

\begin{tabular}{|l|c|l|}
\hline "STATUS" & Número de empresas & \multicolumn{1}{|c|}{ Descrição dos "status" } \\
\hline Ativo & 257 & Toda documentação está em dias com a SEMA. \\
\hline Suspenso & 248 & $\begin{array}{l}\text { São empresas que o registro existe, mas } \\
\text { apresentam alguma irregularidade e não podem } \\
\text { acessar o sistema da SEMA. }\end{array}$ \\
\hline Excluído & 7 & $\begin{array}{l}\text { Tinham seu registro normal, mas foram excluídas } \\
\text { do registro da SEMA por descumprirem alguma } \\
\text { obrigação. }\end{array}$ \\
\hline Reprovado & 16 & $\begin{array}{l}\text { Deram entrada na documentação, mas o registro } \\
\text { não chegou a ser aprovado. }\end{array}$ \\
\hline Suprimido & 28 & $\begin{array}{l}\text { Apresenta o mesmo motivo das que tem o status } \\
\text { reprovado. }\end{array}$ \\
\hline
\end{tabular}

Fonte: Autor

O quadro acima mostra o número de empresas e seu respectivo "status", no qual é possível observar que mais da metade está com o "status" ativo, porém as empresas suspensas representam um montante significativo (248). Dessa forma, caso haja a regularização das empresas com o "status" suspenso, esse montante aumentaria (505) o número de empresas do setor madeireiro na RMB.

Porém, como o objetivo do estudo é trabalhar com empresas que potencialmente geram resíduos, foi feita uma nova filtragem dos dados. Com isso, foram excluídas, dentro das que apresentaram "status" ativo, as que armazenam produtos acabados ou os comercializam, ou seja, não produzem resíduos madeireiros, restando apenas aquelas que processam ou beneficiam a madeira, gerando assim resíduos madeireiros. Dessa forma, das 257 empresas ativas registradas na SEMA, restaram 116 empresas potencialmente geradoras de resíduos, que incluem serrarias, laminadoras, as que beneficiam e as moveleiras, todas localizadas na RMB (Figura 1).

$\mathrm{Na}$ figura, é possível verificar o montante de empresas localizadas apenas na RMB. Porém, esses números devem ser bem maiores, visto que só foram quantificadas as empresas registradas na SEMA com "status" ativos, ou seja, as empresas que não estão registradas ou com o registro suspenso ou cancelados não foram quantificadas. 
Figura 1 - Número de empresas madeireiras ativas que processam ou beneficiam a madeira registradas na SEMA e localizadas na RMB, 2014.

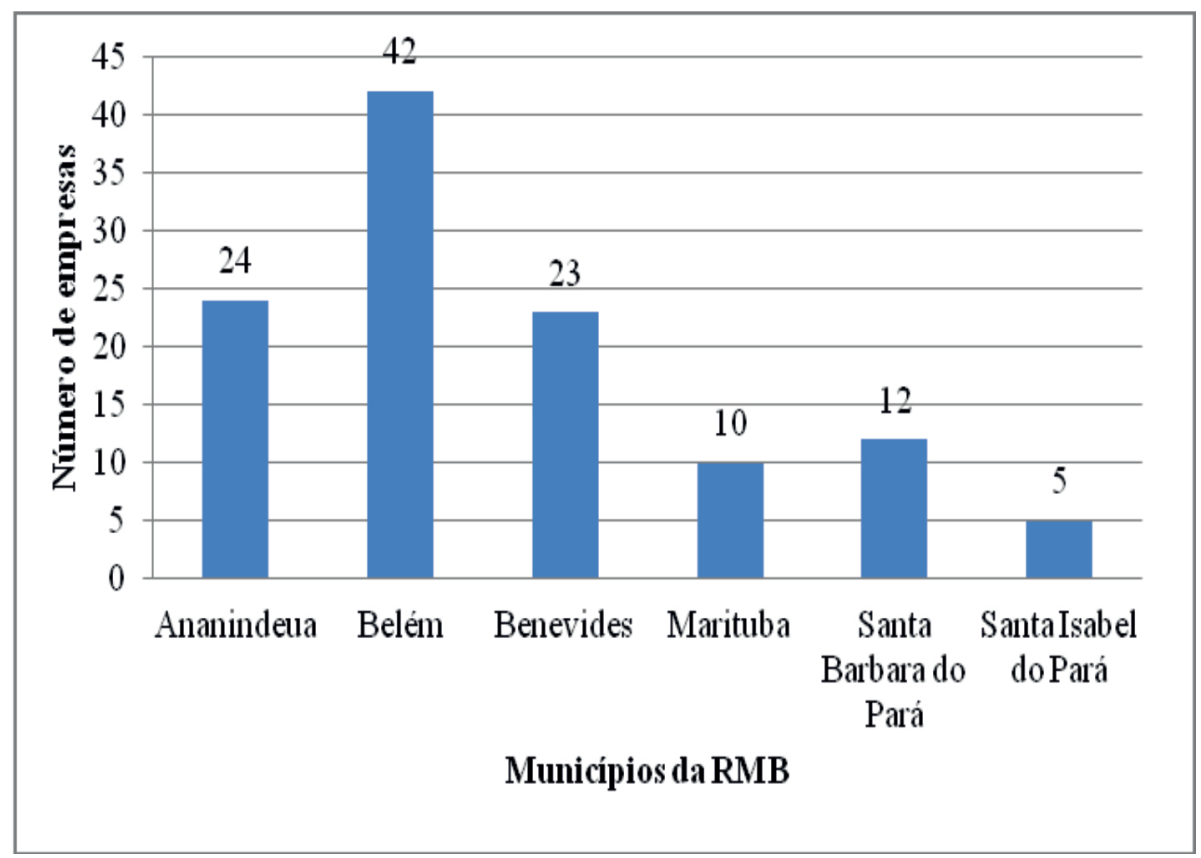

Fonte: Autor

Por outro lado, as informações coletadas no banco de dados da AIMEX foram em número bem menor do que aquelas coletadas na SEMA e mostram uma população de dezessete empresas dividas em quatro municípios da RMB (Figura 2).

A figura mostra que as empresas estão predominantemente instaladas em Belém e Ananindeua devido a sua infraestrutura rodoviária, hidroviária e por serem polos madeireiros consolidados os quais são municípios cujo volume de madeira em tora extraído e consumido é igual ou superior a 100 mil metros cúbicos por ano (VERÍSSIMO et al., 2002).

Ne sse contexto, o município de Belém, juntamente com Ananindeua, Benevides, Marituba e Santa Bárbara do Pará, faz parte de um dos vários polos madeireiros existentes na Amazônia Legal, com um consumo superior a 697 mil $\mathrm{m}^{3}$ em tora por ano, gerando assim aproximadamente 13 mil empregos diretos e indiretos em 2009 (SFB; IMAZON, 2010). 
Figura 2 - Número de empresas madeireiras associadas à AIMEX que atuam na RMB, 2014.

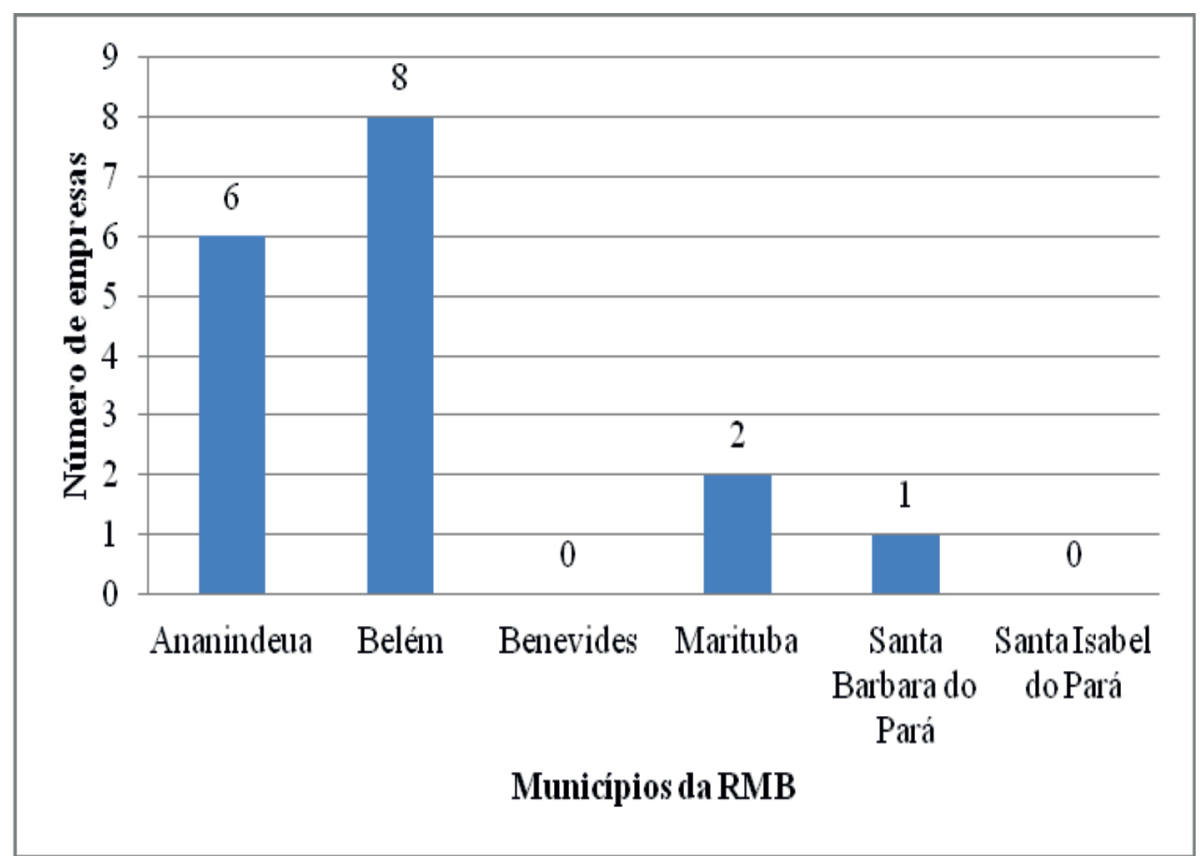

Fonte: Autor

A partir do cruzamento das informações dos dados coletados na AIMEX e na SEMA, verificou-se q ue, das 17 empresas constantes no site da AIME X, onze constavam na lista fornecida pela SEMA. Das seis empresas restantes, duas estavam com "status" suspenso e uma reprovado e, portanto, não constam na análise deste estudo. Com o cruzamento dessas informações, chegou-se ao total de 120 empresas potencialmente geradoras de resíduos madeireiros localizadas na RMB (Figura 3).

Em termos quantitativos, por município, quando se considera os dados disponibilizados pela SEMA (2014), é possível perceber que Belém possui o maior número de empresas madeireiras e Santa Isabel do Par á, o menor.

Nessa perspectiva, Belém possui várias madeireiras ao longo de sua orla até o Distrito de Icoaraci e sua relação com este tipo de indústria se dá em virtude das facilidades de aporte de embarcações e comercialização de madeiras, já que a maior parte das madeireiras sempre ocuparam e ainda ocupam a sua orla; fato que vem mudando com o processo de modernização e urbanização da capital paraense; enquanto o município de Ananindeua possui um Distrito Industrial e, portanto, grande parte das indústrias madeireiras encontram-se localizadas nesse 
polo. As facilidades de locomoção pelos rios e o fato de serem as duas maiores cidades da zona metropolitana, incluindo a capital (Belém), também explicam o porquê destas duas localidades serem atraídas de maneira expressiva por uma grande quantidade de empresas de matriz florestal.

Figura 3 - Número de empresas madeireiras ativas que processam ou beneficiam associadas à AIMEX e registradas na SEMA em 2014.

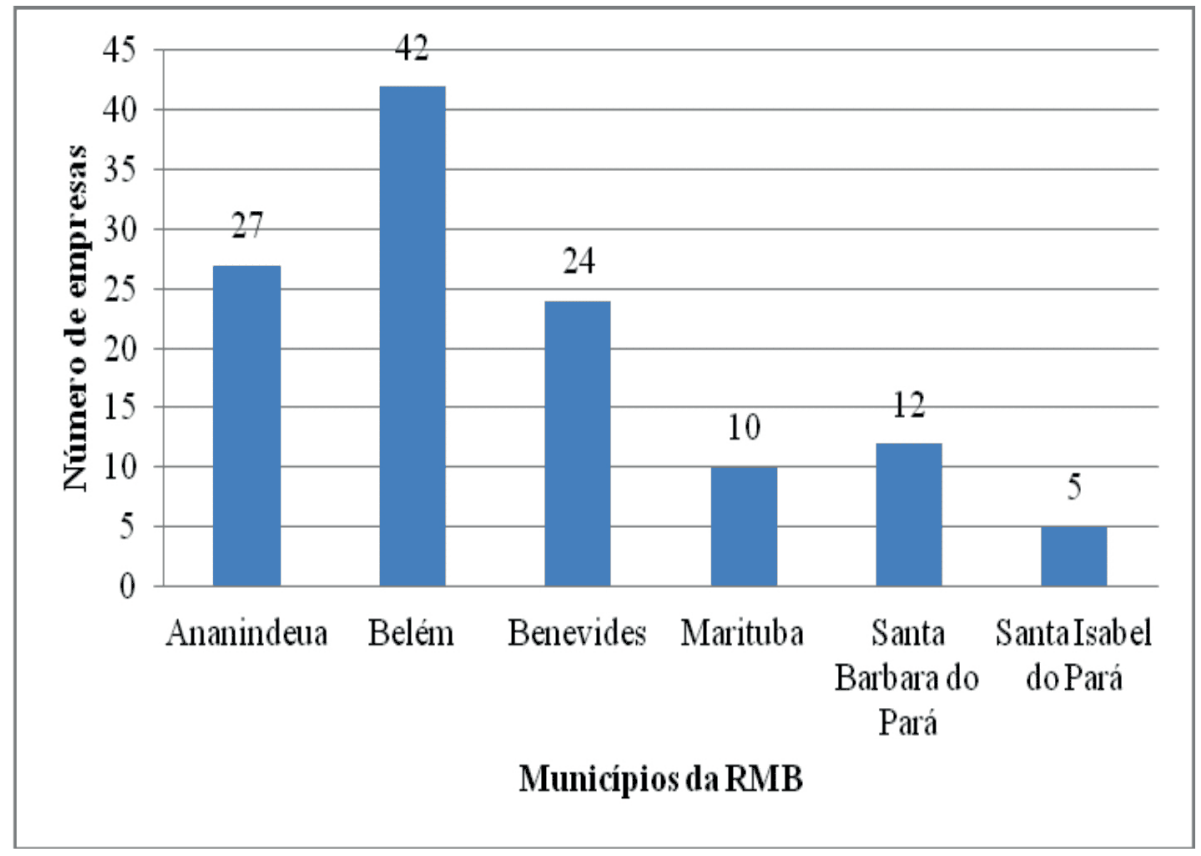

Fonte: Autor

As informações preliminares apontam que o número de empresas com potencial gerador de resíduos pode estar subestimado, o que pode dificultar os trabalhos de fiscalização; tal fato interfere, diretamente, na formulação de políticas públicas direcionadas a essa questão. Uma maior e melhor interação e compartilhamento das informações entre os órgãos públicos e privados que tratam da problemática das madeireiras no Pará também é aconselhável, visto que tais informações são imprescindíveis para a tomada de decisões nas mais diferentes esferas públicas, no sentido de implementar ações que visem à gestão dos resíduos sólidos provenientes da indústria madeireira na Região Metropolitana de Belém, a fim de que não causem danos ambientais e sociais. 


\section{REFERÊNCIAS}

BATISTA, D. C. et al. Desempenho de uma serraria com base na eficiência e na amostragem do trabalho. Floresta Ambiente, v. 20, n. 2, p. 271-280. 2013.

CERQUEIRA, P. H. A. Análise dos Resíduos Madeireiros Gerados Pelas Serrarias do Município de Eunápolis. Floresta Ambiente, v. 19, n. 4, p. 506-510. 2012

FERREIRA, L. V. et al. O desmatamento na Amazônia e a importância das áreas protegidas. Estudos Avançados, v. 19, n. 53, p. 157-166. 2005.

HASAN, A. R.; GABRIELE, H. S.; TOWNSEND. Online sorting of recovered wood waste by automated XRF-technology: Part II. Sorting efficiencies. Elsevier / Waste Management, v. 31, n. 4, p. 695-704. 2011.

LELIS, A.T. Biodeterioração de madeiras em edificações. São Paulo: Instituto de Pesquisas Tecnológicas, 2001. 54p.

RIUL, M.; RIBEIRO E. L. Diagnóstico e Diretrizes para a Gestão de Resíduos no APL de Móveis de João Pessoa-PB. UNOPAR Cient. Exatas Tecnol., Londrina, v. 11, n. 1, p. 15-24, Nov. 2012.

SERVIÇO FLORESTAL BRASILEIRO - SFB e INSTITUTO DO HOMEM E MEIO AMBIENTE DA AMAZÔNIA - IMAZON. A atividade madeireira na Amazônia brasileira: produção, receita e mercados. Belém, PA: Serviço Florestal Brasileiro (SFB); Instituto do Homem e Meio Ambiente da Amazônia (Imazon), 2010. 32p.

VERÍSSIMO, A. et al. Áreas para produção florestal manejada: Detalhamento do Macrozoneamento Ecológico Econômico do Estado do Pará. Belém, PA: Instituto do Homem e Meio Ambiente da Amazônia (Imazon), 2006. 82p.

VERÍSSIMO, A. et al. Polos Madeireiros do Estado do Pará. Belém, PA: Instituto do Homem e Meio Ambiente da Amazônia (Imazon), 2002. 75p.

ZENID, G. J. Madeira: Uso sustentável na construção civil. 2ed. São Paulo: Instituto de Pesquisas Tecnológicas, SVMA, 2009. 99p 
